\title{
Exploring the causes of adverse events in hospitals and potential prevention strategies
}

\author{
M Smits, ${ }^{1}$ M Zegers, ${ }^{1}$ P P Groenewegen, ${ }^{1,2}$ D R M Timmermans, ${ }^{3}$ L Zwaan, ${ }^{3}$ \\ $\mathrm{G}$ van der Wal, ${ }^{3} \mathrm{C}$ Wagner ${ }^{1,3}$
}

${ }^{1}$ NIVEL, Netherlands Institute for Health Services Research, Utrecht, The Netherlands ${ }^{2}$ Department of Sociology and Department of Human Geography, Utrecht University, Utrecht, The Netherlands ${ }^{3}$ EMGO Institute, Department of Public and Occupational Health, VU University Medical Center, Amsterdam, The Netherlands

\section{Correspondence to} Marleen Smits, NIVEL, Netherlands Institute for Health Services Research, PO Box 1568, 3500 BN Utrecht, The Netherlands; m.smits@nivel.nl

Accepted 6 March 2009 Published Online First 8 February 2010

\section{ABSTRACT}

Objectives To examine the causes of adverse events (AEs) and potential prevention strategies to minimise the occurrence of AEs in hospitalised patients.

Methods For the 744 AEs identified in the patient record review study in 21 Dutch hospitals, trained reviewers

were asked to select all causal factors that contributed to the AE. The results were analysed together with data on preventability and consequences of AEs. In addition, the reviewers selected one or more prevention strategies for each preventable AE. The recommended prevention strategies were analysed together with four general causal categories: technical, human, organisational and patient-related factors.

Results Human causes were predominantly involved in the causation of $A E s$ (in $61 \%$ of the AEs), $61 \%$ of those being preventable and $13 \%$ leading to permanent disability. In $39 \%$ of the AEs, patient-related factors were involved, in $14 \%$ organisational factors and in $4 \%$ technical factors. Organisational causes contributed relatively often to preventable AEs (93\%) and AEs resulting in permanent disability (20\%). Recommended strategies to prevent AEs were quality assurance/peer review, evaluation of safety behaviour, training and procedures. For the AEs with human and patient-related causes, reviewers predominantly recommended quality assurance/peer review. AEs caused by organisational factors were considered preventable by improving procedures.

Discussion Healthcare interventions directed at human causes are recommended because these play a large role in AE causation. In addition, it seems worthwhile to direct interventions on organisational causes because the AEs they cause are nearly always believed to be preventable. Organisational factors are thus relatively easy to tackle. Future research designs should allow researchers to interview healthcare providers that were involved in the event, as an additional source of information on contributing factors.

Several studies in various countries have shown that a substantial number of patients experience adverse events (AEs) in hospitals. ${ }^{1-9}$ These studies have reported $\mathrm{AE}$ incidence rates ranging from $2.9 \%$ to $16.6 \%$ of all hospital admissions. Of the AEs, $25 \%$ to $50 \%$ were considered preventable, and in $5 \%$ to $13 \%$ of the AEs the patient died. The studies on the incidence of AEs have enlarged the sense of urgency to take effective countermeasures to increase patient safety in hospitals. Reduction of AEs can only be achieved if these interventions tackle the dominant underlying causes. To explore the causes of AEs and to gain more insight into potential prevention strategies, we carried out additional analyses of data that were gathered in the Dutch patient record review study. ${ }^{9} 10$

In accordance with the theoretical framework of Reason, ${ }^{11}$ the focus of our study was on active human behavioural failures and on latent factors present within the system (organisational and technical factors) before an accident sequence involving human behaviour actually begins. In other words, healthcare providers' behaviour was not the single point of attention; the conditions under which they work were considered important as well-for example, the design of equipment that healthcare providers have to work with or the number of staff installed by hospital management.

When planning quality and patient safety interventions in hospitals, information about prevention strategies that tackle the causal factors to prevent future AEs is valuable. Perhaps the most effective strategies to reduce AEs are improving technology, optimising procedures or training programmes for healthcare providers. Therefore, the reviewers in our study assessed potential prevention strategies next to the causes of the AEs. This information can be an aid for policy-makers in healthcare.

The objectives of our study were to gain more insight into (a) the causes of AEs, (b) the relationship between the causes of AEs and the preventability and health consequences of the AEs, (c) potential prevention strategies to prevent AEs and (d) the relevance of the prevention strategies for each main causal factor type.

\section{METHODS}

\section{Study design and setting}

A retrospective patient record review study was conducted to examine AEs in Dutch hospitals with a stratified random sample of 21 hospitals: 4 university, 6 tertiary teaching and 11 general hospitals. From each hospital, a stratified random sample was selected of 200 admissions of patients discharged from the hospital in 2004 (>24 h stay) and 200 (or less if the total of patients who died in 2004 was lower) admissions of patients deceased in the hospital in 2004, excluding admissions of psychiatry, obstetrics and children $<1$ year old. Admissions of deceased patients and patients admitted to university hospitals were oversampled. The methods of determining AEs were based on previous AE studies in other countries and have been described in detail elsewhere. ${ }^{10}$

\section{Structured review of patient records}

Between August 2005 and October 2006, 55 trained physicians reviewed the medical, nursing and, if 
available, outpatient record of all sampled admissions that contained triggers (clues) for AEs-for example, an unplanned readmission, unplanned return to the operating room or unexpected death. The presence of one or more of the 18 predefined triggers was judged in advance by trained nurses. For each patient record, two physician reviewers determined independently the presence, consequences and degree of preventability of the AEs, based on a standardised procedure and review form. An $A E$ was defined as an unintended injury among hospitalised patients that results in disability, death or prolonged hospital stay, and was caused by healthcare management. Preventable refers to care that fell below the current level of expected performance for practitioners or systems. Disability was defined as temporary or permanent impairment of physical or mental function attributable to the AE (including prolonged or strengthened treatment, readmission and death). ${ }^{10}$

In total, 7926 patient records were reviewed. The reviewers identified $744 \mathrm{AEs}$ in the records. When generalised to all hospital admissions, the $\mathrm{AE}$ incidence rate was $5.7 \%$ (corrected for overrepresentation of patients admitted to a university hospital and patients who died in hospital). Forty per cent of the AEs were considered preventable and $12.8 \%$ resulted in permanent disability (including death). Detailed results of this study are reported in a previous publication. ${ }^{9}$

Part of the review procedure was an assessment of the underlying causes and prevention strategies of each AE as judged by the physician reviewers. The review form presented five main causal factor categories to choose from: technical, human, organisational, patient-related and other. For each AE, reviewers selected one or more of these main causal factors based on the information in the medical record and their perception of the situation. Having opted technical, organisational and/or human causes, reviewers selected one or more subcategories of causal factors within these main categories. The causal factor categories were derived from a recognised taxonomy of root causes: the Eindhoven Classification Model (ECM) of PRISMA-Medical, a root cause analysis tool. ${ }^{12} 13$ The taxonomy includes concepts of the theoretical frameworks of Reason ${ }^{11}$ and Rasmussen. ${ }^{14}$ Reason's system approach to accident causation can be seen in the recognition of latent factors (organisational and technical) next to active failures (human). The human categories of the ECM fit in the skill-rules-knowledge framework (SRK framework) of Rasmussen (see Box 1).

Reason used the SRK framework for his Model of Unsafe Acts, in which "violation" is added to the basic error types of the SRK framework. ${ }^{11}$ Violations are deliberate deviations from rules or procedures. The ECM does not cover the concept of violations. We have added violations to the taxonomy to cover all concepts of the Model of Unsafe Acts. In table 1, the main causal factor categories and subcategories are listed and explained.

Beside the causal factor categories, the review form distinguished 10 prevention strategies (table 2). The categories were based on PRISMA ${ }^{13}$ and the Canadian patient record review study. ${ }^{7}$ For each AE, the reviewers could select one or more prevention strategies that they judged relevant for the AE.

There were two reviewers per AE. Both reviewers could select more than one cause and more than one prevention strategy per AE. We reported all causes and prevention strategies that were selected by this panel of two physicians irrespective of whether one or both reviewers selected the particular cause or prevention strategy. We wanted to find a broad variety of indications to improve patient safety in our exploratory study and we did not intend the reviewers to reach a shared conclusion. Because there is a lot of information in patient records, the likelihood of
Box 1 Skill-based, rules-based and knowledge-based behaviour $^{14}$

To understand the mechanisms behind human error, Rasmussen developed the skill-rules-knowledge framework (SRK framework), with the notion that human behaviour can be controlled at different levels of conscious control, dependent on the degree of familiarity with the task and the environment. At the skill-based level, behaviour is regulated by the lowest level of conscious involvement and is characteristic of highly routinised and automated activities. The individual is seldom able to verbalise how performance of the behaviour is controlled or explain the information on which the performance is based. Rule-based behaviour requires some degree of conscious involvement; at this level, behaviour is controlled by stored rules derived from experience or other's know-how. At this level, performance is typically based on specific ability and the rules for performance can be verbalised. At the knowledge-based level, stored rules do no longer apply and a novel situation is presented for which a plan must be developed to solve a problem. Here, the individual uses conceptual reasoning or trial-and-error.

missing a causal factor (false negative) will be larger than the likelihood that a causal factor is selected mistakenly when in reality it does not exist (false positive). Thus, reviewers were considered complementary to each other.

When both reviewers selected the same causal factor or prevention strategy for an $\mathrm{AE}$, these were counted only once in the analyses.

\section{Statistical analysis}

Causes of AEs and prevention strategies were analysed using descriptive statistics and frequency tables. Frequencies were calculated using weights that corrected for overrepresentation of patients admitted to a university hospital and for overrepresentation of patients who died in hospital. The sampling weight was the inverse of the probability of being included in the sample owing to the sampling design. ${ }^{9}$

For 8 of the 744 AEs, neither of the reviewers filled out any of the items in the review form about causal factors. Consequently, results on causal factors will be presented for 736 AEs. For the prevention strategies, results of the 315 AEs that were considered preventable will be presented. All statistical analyses were performed using SPSS V.14.0.

\section{RESULTS}

\section{Causes of AEs (objectives a and $\mathbf{b}$ )}

The 736 AEs were associated with 1017 main causal factors because reviewers could select more than one category per AE. Figure 1 presents the weighted percentages of the five main causal factor categories. Human causes were predominantly involved in AE causation (in $61 \%$ of the AEs). In $39 \%$ of the AEs, patient-related factors were involved. In $14 \%$ of the AEs, organisational factors contributed to the $\mathrm{AE}$, in $4 \%$ technical factors and in $19 \%$ other factors.

Organisational factors had a relatively high proportion of AEs that were preventable (out of all AEs with an organisational cause involved, 93\% was considered preventable), followed by human causes (61\% of all AEs with human causes). Organisational factors also had a relatively high proportion of AEs that led to permanent disability (20\%). Technical factors had low proportions of preventable AEs (22\%) and AEs resulting in 
Table 1 Explanation of causal factors categories

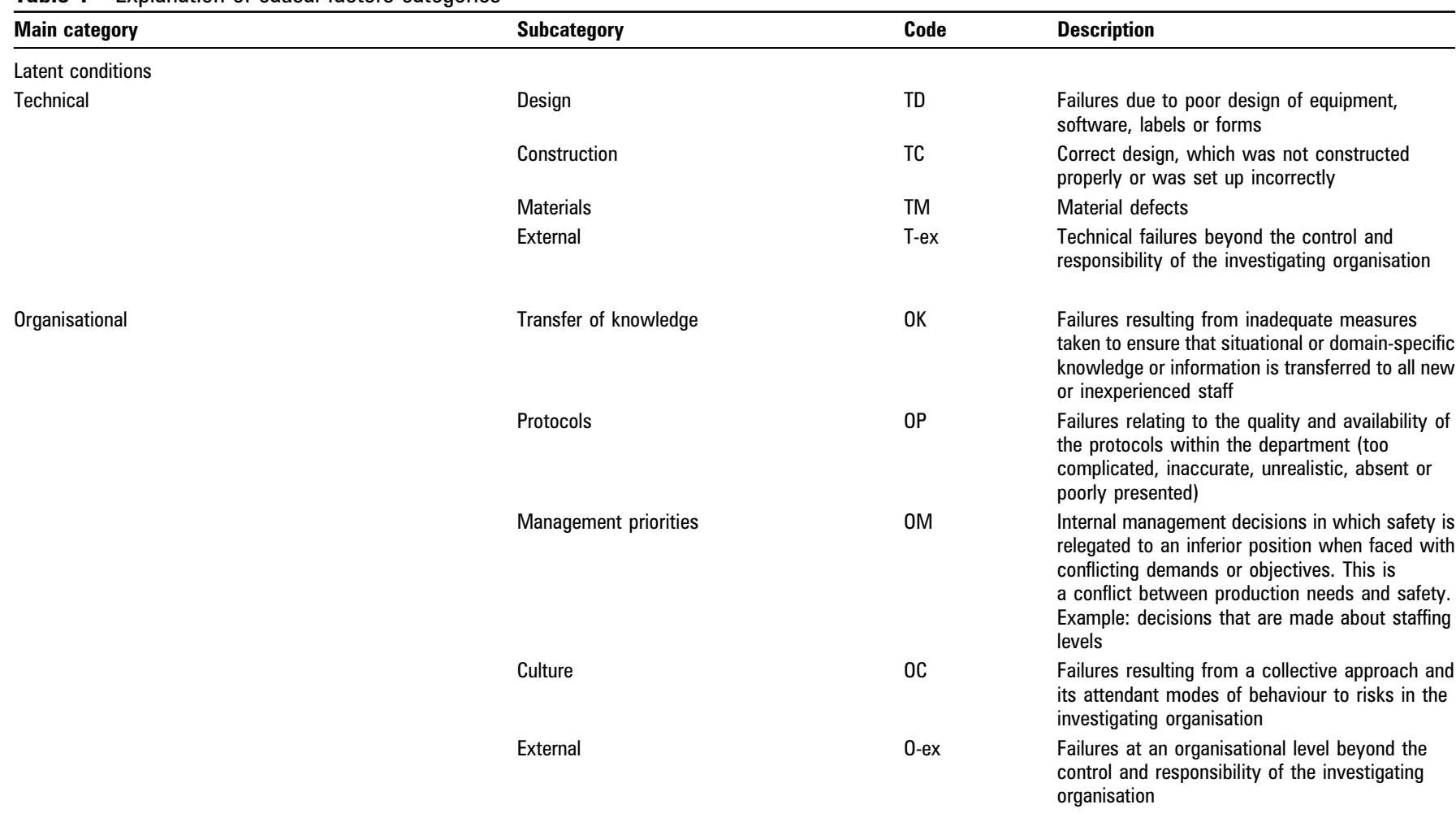

Active errors

Human

Knowledge-based behaviour

Rule-based behaviour

Verification

HRV

Intervention

Monitoring

HRM

Skill-based behaviour

Slips

HSS

Tripping

HST

External

H-ex

Violations

Violations

V

Other factors

Patient related
Patient-related factor

PRF

Unclassifiable
HKK

HRC

Other
The inability of an individual to apply his/her existing knowledge to a novel situation

An incorrect fit between an individuals training or education and a particular task

A lack of task coordination within a healthcare team in an organisation. Example: an essential task not being performed because everyone thought that someone else had completed the task

The correct and complete assessment of a situation including related conditions of the patient and materials to be used before starting the intervention

Failures that result from faulty task planning and execution. Example: washing red cells by the same protocol as platelets

Monitoring a process or patient status. Example: a trained technologist operating an automated instrument and not realising that a pipette that dispenses reagents is clogged

Failures in performance of highly developed skills. Example: a computer entry error

Failures in whole body movements. These errors are often referred to as "slipping, tripping or falling". Examples: a blood bag slipping out of one's hands and breaking or tripping over a loose tile on the floor

Human failures originating beyond the control and responsibility of the investigating organisation

Failures by deliberate deviations from rules or procedures

Failures related to patient characteristics or conditions, which are beyond the control of staff and influence treatment. Example: communicative skills, treatment compliance

Failures that cannot be classified in any other category-eg, complication, abstain policy, rare disease 
Table 2 Explanation of prevention strategy categories

\begin{tabular}{ll}
\hline Prevention strategies & Description \\
\hline Technology/equipment & Redesigning of hardware, software or interface parts of the man-machine system \\
Procedures & Completing or improving formal and informal procedures \\
Information and communication & Completing or improving available sources of information and communication structures \\
Training & Improving (re)training programmes for skills needed \\
Motivation & Increasing the level of voluntary obedience to generally accepted rules by applying principles of positive behaviour modification \\
Up-scaling & Handling the problem at a higher organisational level-eg, hospital department or hospital management level \\
Evaluation & Evaluating the current way of behaving regarding safety \\
Quality assurance/peer review & Continuously monitoring of data quality based on prespecified standards and assessment of a health professional's performance \\
Financial investment & by one or more individuals in the same field \\
Personnel & Financial investments in required areas-eg, increasing the availability of facilities and equipment \\
\hline
\end{tabular}

Descriptions of first six prevention strategies derived from Van der Schaaf and Habraken. ${ }^{13}$

permanent disability (5\%) (figure 1). In the subgroup of AEs that were preventable and led to permanent disability, the distribution of causal factors shows that in these AEs there were nearly always human causes involved (94\%), frequently organisational $(36 \%)$ and patient-related causes (33\%), and rarely technical causes (1\%) (figure 2 ).

For the technical, human and organisational categories, one or more specific subcategories of causes were selected as well. The results are presented in table 3 (see also Appendix for examples). Material defects were the most common technical factors (34\% of all AEs with technical causes). Contrary to the other technical factors, material defects were all associated with preventable AEs.

Human causes were predominantly knowledge based (46\% of all AEs with human causes) and/or related to rule-based failures regarding monitoring (27\%), intervention (26\%) and/or verification $(21 \%)$. These causal factors, as well as violations, were associated with large proportions of preventable AEs (74\% to $89 \%$ ).

Transfer of knowledge was the most frequent organisational factor contributing to AEs (50\% of all AEs with organisational causes). Failures relating to the quality and availability of protocols were related to the highest proportion of AEs with permanent disability (33\%).

\section{Potential prevention strategies (objectives $c$ and d)}

For all preventable AEs $(n=315)$, reviewers selected 871 prevention strategies (table 4). Quality assurance/peer review was the most frequently selected strategy (65\%), followed by evaluation $(53 \%)$ training $(50 \%)$ and procedures $(40 \%)$. Financial investments, up-scaling of the problem to a higher organisational level and increasing the number of personnel were least often considered as relevant methods to prevent AEs ( $2 \%$ to $3 \%$ ).

For the AEs with human and patient-related causes, reviewers predominantly recommended quality assurance/peer review $(68 \%$ and $78 \%$, respectively). AEs caused by organisational factors were considered preventable by improving procedures (67\%).

\section{DISCUSSION \\ General findings}

The study provides an initial overview of causes of AEs and required remedial action. The causes identified by the reviewers were predominantly related to human errors, especially knowledge-based and rule-based errors-for example, incorrect reasoning by a healthcare provider or not verifying if all necessary instruments were present before the start of a procedure. AEs with organisational factors involved were relatively often preventable and resulted relatively often in permanent disability, particularly AEs caused by inadequate or unavailable protocols. Overall, technical factors (eg, an inadequate construction of equipment) contributed relatively less often to preventable AEs and AEs resulting in permanent disability. However, the technical factors within the material defects subgroup all contributed to preventable AEs.

Distribution of main causes of AEs: proportions of preventable AEs (top graph) and AEs leading to permanent disability (including death) (bottom graph).
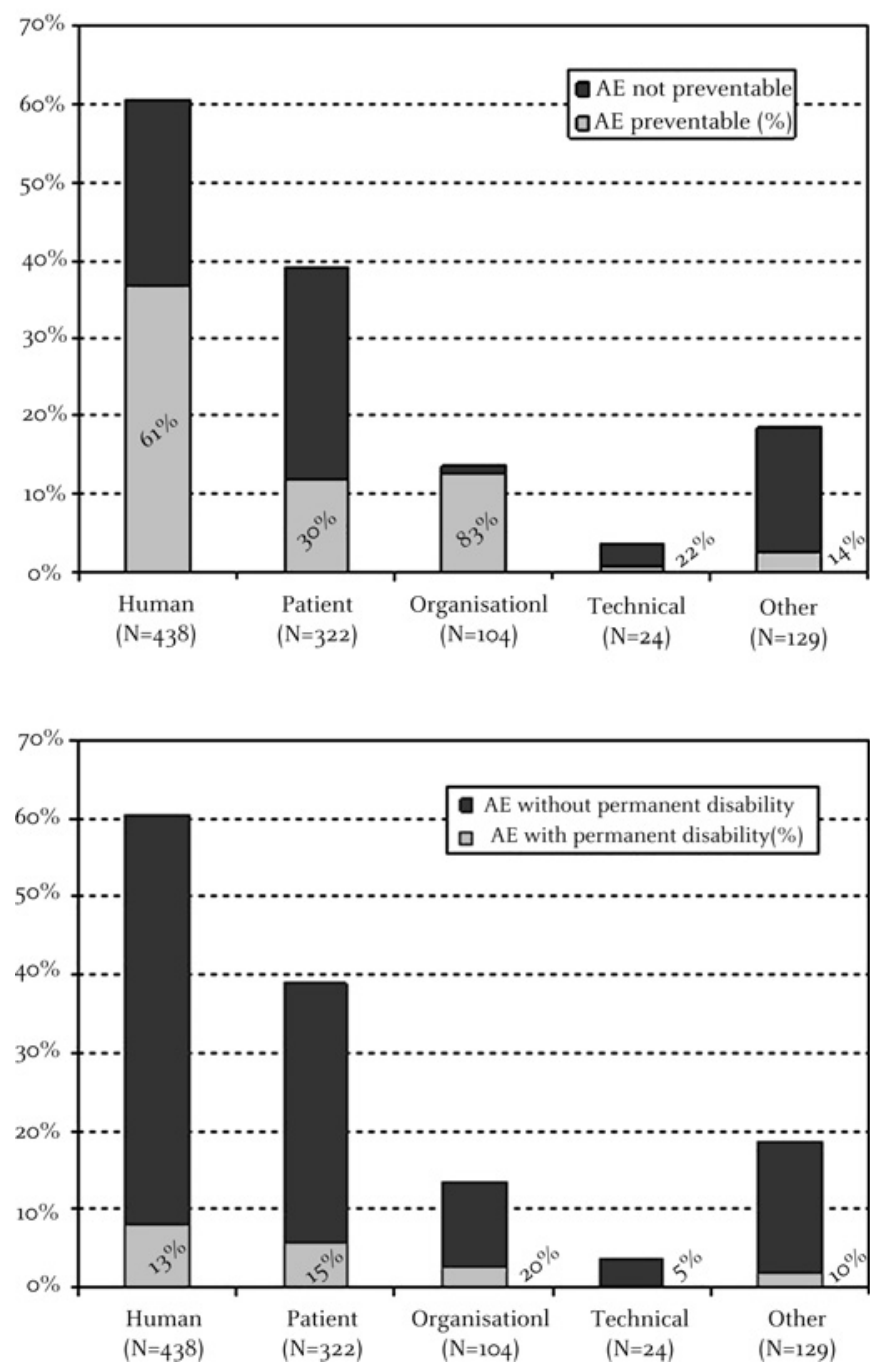

Figure 1 Percentages are weighted for over-sampling of deceased patients and patients admitted to a university hospital (N: actual numbers of causes, not weighted). Reviewers could select more than one casual factor per AE. Preventability is related to the assessment of the preventability of the $A E$, not the preventability of each cause underlying the $A E$. 
Distribution of main causes of preventable AEs that lead to permanent disability (including death).

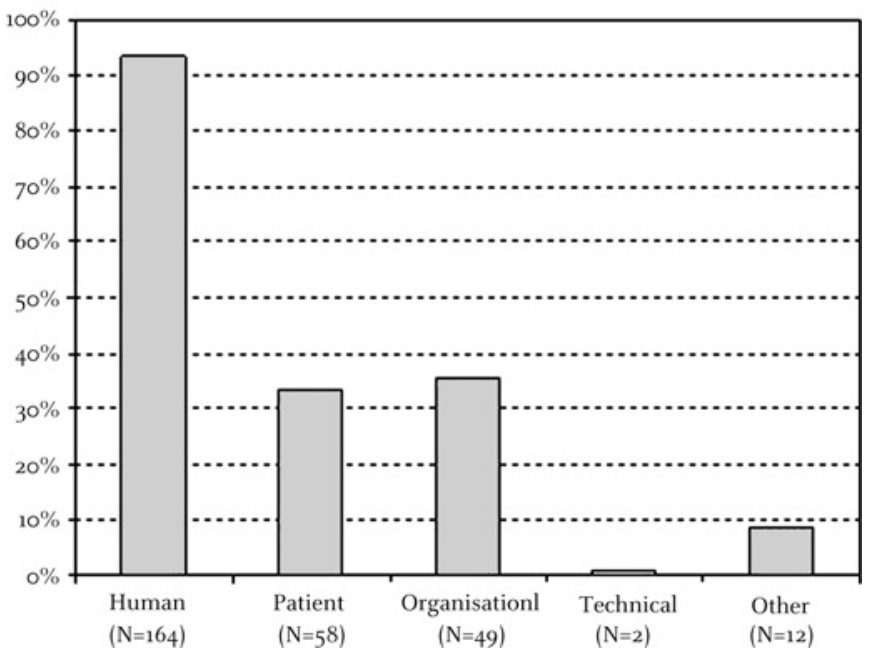

Figure 2 Percentages are weighted for over-sampling of deceased patients and patients admitted to a university hospital ( $\mathrm{N}$ : actual numbers of causes, not weighted). Reviewers could select more than one casual factor per AE. Preventability is related to the assessment of the preventability of the $A E$, not the preventability of each cause underlying the $\mathrm{AE}$.

The most frequently recommended strategies to prevent AEs were quality assurance/peer review, evaluation of safety behaviour, training and improvement of procedures. For AEs with human and patient-related causes, reviewers indicated these could be prevented by quality assurance/peer review. For AEs caused by organisational factors, reviewers predominantly recommended completing or improving procedures.

To sum up, active failures played a large role in AE causation, but the reviewers also identified latent factors that were present in the system and that contributed to AEs. To tackle active human causes, which occurred most frequently, quality assurance/peer review was recommended. To deal with latent organisational factors, which underlie AEs that were believed to be most preventable and were associated with the most severe consequences, improving procedures was recommended.

\section{Comparison with previous record review studies}

Up to now, two retrospective patient record review studies have assessed the causes of the $\mathrm{AEs}^{15}{ }^{16}$ and two have examined possible prevention strategies. ${ }^{15}{ }^{17}$ It is quite difficult to compare our results with these and other studies on the causes and prevention strategies of AEs because the classifications used vary to a large extent and most studies examined the causes of a specific group of AEs, such as medication errors. However, the design of the Australian record review study was comparable to ours. ${ }^{215}$ Wilson et al ${ }^{2}$ reexamined the 2353 AEs identified earlier and found that AEs in which the cause was a cognitive failure were associated with high preventability scores. This is similar to our findings: we found that AEs caused by human errors were often preventable, next to AEs caused by organisational factors. Latent factors (organisational and technical factors) were not examined in the Australian study. Furthermore, the main prevention strategies were comparable to our results: they recommended new or better implemented policies and protocols, better formal quality monitoring and better education. ${ }^{15}$

\section{Strengths and limitations}

Our study has some advantages over previous studies on the causes of AEs. Causes and prevention strategies were examined directly, together with the assessment of the presence of AEs, instead of afterwards. The reviews were thus based on original records and not on summaries of information compiled for the review, like in the Australian study. ${ }^{15}$ Moreover, we used a classification of causes in five main categories, divided in several subcategories. The categories were based on the well-established

Table 3 Causes of AEs (subcategories): proportions with preventable AEs and AEs leading to permanent disability (including death)

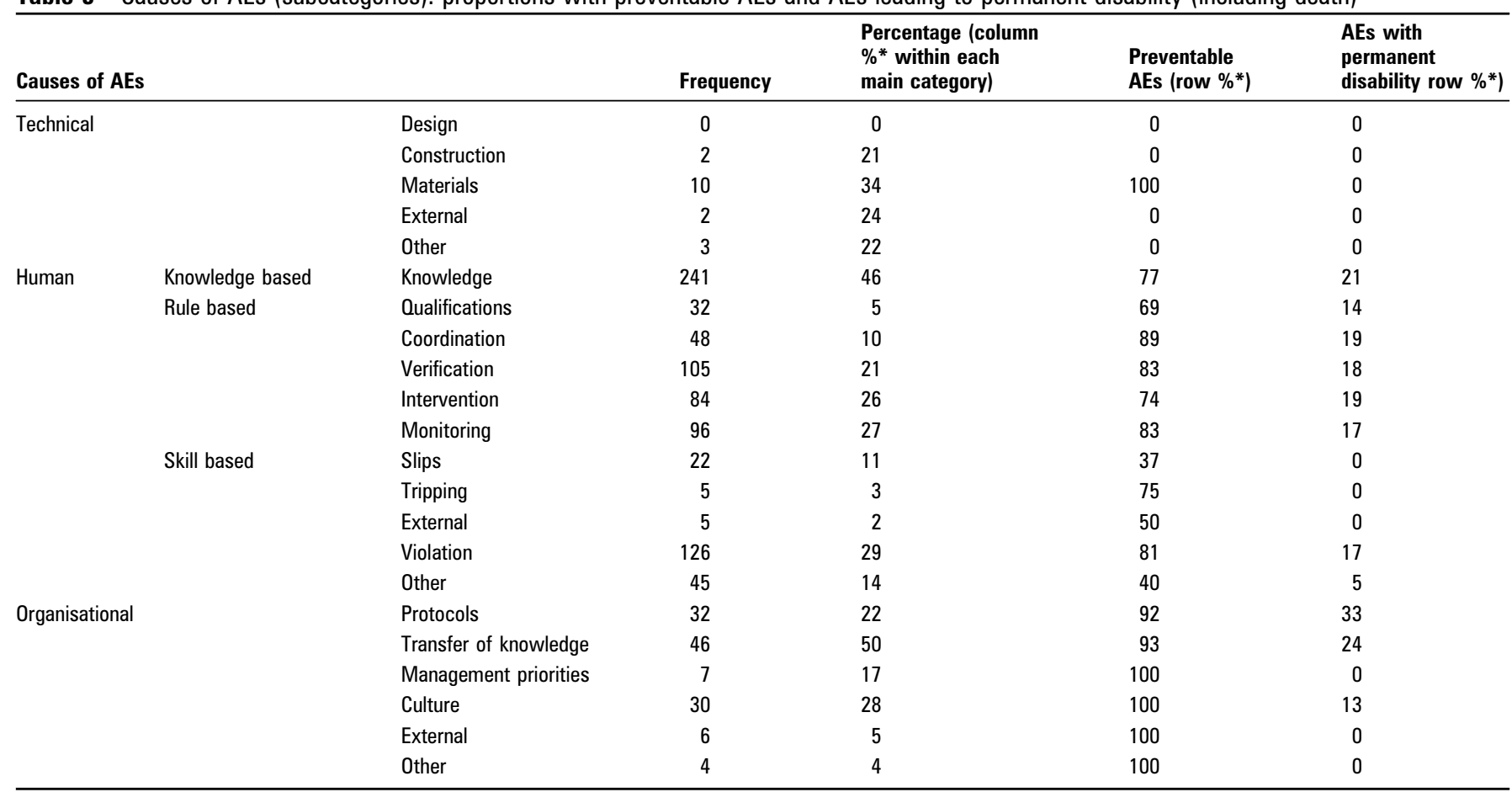

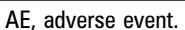

Reviewers could select more than one causal factor per AE. Preventability is related to the assessment of the preventability of the AE, not to the preventability of each cause underlying the AE. *Percentages are weighted for oversampling of deceased patients and of patients admitted to a university hospital. 
Table 4 Recommended strategies for preventing AEs in relation to causes of AEs

\begin{tabular}{|c|c|c|c|c|c|c|}
\hline Prevention strategies & $\begin{array}{l}\text { Frequency } \\
n=315\end{array}$ & $\begin{array}{l}\text { Percentage* } \\
\mathrm{n}=315\end{array}$ & $\begin{array}{l}\text { Human } \dagger \\
\text { (column \%*) } \\
n=292\end{array}$ & $\begin{array}{l}\text { Organisational } \dagger \\
\text { (column \%*) } \\
\mathbf{n}=92\end{array}$ & $\begin{array}{l}\text { Patient related } \dagger \\
\text { (column } \% * \text { ) } \\
n=100\end{array}$ & $\begin{array}{l}\text { Other } \dagger \\
\text { (column \%*) } \\
n=21\end{array}$ \\
\hline Quality assurance/peer review & 199 & 65 & 68 & 54 & 78 & 67 \\
\hline Training & 136 & 50 & 53 & 36 & 48 & 31 \\
\hline Procedures & 138 & 40 & 37 & 67 & 53 & 39 \\
\hline Motivation & 74 & 28 & 28 & 46 & 37 & 15 \\
\hline Personnel & 9 & 3 & 3 & 3 & 2 & 0 \\
\hline Up-scaling & 6 & 2 & 2 & 3 & 3 & 0 \\
\hline Financial investment & 5 & 2 & 1 & 3 & 3 & 0 \\
\hline Other & 21 & 4 & 4 & 5 & 2 & 0 \\
\hline
\end{tabular}

Reviewers could select more than one prevention strategy per AE. The number of preventable AEs with technical causes was too small to calculate percentages for the prevention strategy categories.

*Percentages were weighted for oversampling of deceased patients and of patients admitted to a university hospital.

†Within subgroups of AEs that have human, organisational, patient-related or other causes, respectively.

theoretical frameworks of Reason and Rasmussen, which are often referred to in error management and patient safety literature.

There are however some limitations. The usability of a patient record as a means to examine the causes of AEs is limited. It is hard to get information about all contributing factors by reading the patient record only. Human actions are regularly reported on in a patient record and therefore human causes are most visible for reviewers. One can imagine that healthcare providers are less inclined to note technical or organisational factors in the medical or nursing record of an individual patient. This can be an explanation for the relatively small amounts of technical and organisational factors found in our study.

Another limitation is that there was no contact between the reviewers and the involved healthcare professionals to gather more information about the event and contributing factors. This was not possible because of privacy restrictions made before the study started and because the reviews were performed 1 to 2 years after the occurrence of the AE. Neale et a $1^{18}$ attempted to overcome these difficulties in their pilot study in one UK hospital. The clinical staff of the hospital assessed patient records, shortly after discharge, in combination with external reviewers. Clinical teams have "inside" knowledge about the care provided to the patients. ${ }^{18}$

Despite the limitations, exploratory study has offered an interesting overview of the causes and prevention strategies of AEs.

\section{Implications for practice and future research}

The Dutch Society of Medical Specialists, among others, has formulated a national patient safety action campaign for hospitals "Prevent harm, work safely" in 2008. The results of the present study served as input for this safety programme. The programme includes quality monitoring at department level, (team) training, evidence-based procedures and evaluations of the Individual Functioning of Medical Specialists, including the construction of a personal portfolio, a personal progress plan and annual interviews about quality of care and communication with colleagues and patients. ${ }^{19}$

We recommend that in future research into causes of AEs a more prospective design is used in which the period between the occurrence of the $\mathrm{AE}$ and the assessment of its causes is only a few weeks. In that case, involved healthcare providers can be interviewed to provide more information about contributing factors soon after the occurrence of the $\mathrm{AE}$.
Finally, studies on AEs should use the same taxonomy for causes of AEs to make national and international comparisons of causes of AEs possible. The World Health Organization is formulating a taxonomy of patient safety events and contributing factors. $^{2021}$ This taxonomy is partly based on the ECM we have used in our research.

Acknowledgements We thank everyone who contributed to the study: the physicians who reviewed the patient records, the researchers who coordinated the data collection and the 21 participating hospitals and their staff who facilitated the patient records.

Funding The Dutch Patient Safety Research Program has been initiated by the Dutch Society of Medical Specialists (in Dutch: Orde van Medisch Specialisten) with financial support from the Ministry of Health, Welfare and Sport. The Program is carried out by EMGO Institute/NUmc and NIVEL.

\section{Competing interests None.}

Ethics approval The project and methods had been granted ethical approval by VU University Medical Centre in Amsterdam, The Netherlands.

\section{REFERENCES}

1. Brennan TA, Leape LL, Laird NM, et al. Incidence of adverse events and negligence in hospitalized patients. Results of the Harvard Medical Practice Study I. N Engl J Med 1991;324:370-6.

2. Wilson RM, Runciman WB, Gibberd RW, et al. The quality in Australian health care study. Med J Aust 1995;163:458-71.

3. Thomas EJ, Studdert DM, Burstin HR, et al. Incidence and types of adverse events and negligent care in Utah and Colorado. Med Care 2000;38:261-71.

4. Schioler T, Lipczak H, Pedersen BL, et al. Incidence of adverse events in hospitals. A retrospective study of medical records (in Danish). Ugeskr Laeger 2001;163:5370-8.

5. Vincent C, Neale G, Woloshynowych M. Adverse events in British hospitals: preliminary retrospective record review. BMJ 2001;322:517-19.

6. Davis $\mathbf{P}$, Lay-Yee R, Briant R, et al. Adverse events in New Zealand public hospitals 1 occurrence and impact. N Z Med J 2002;115:U271.

7. Baker GR, Norton PG, Flintoft V, et al. The Canadian Adverse Events Study: the incidence of adverse events among hospital patients in Canada. CMAJ 2004:170:1678-86

8. Michel P, Quenon JL, De Sarasqueta AM, et al. Comparison of three methods for estimating rates of adverse events and rates of preventable adverse events in acute care hospitals. BMJ 2004;328:199.

9. Zegers M, Bruijne MC de, Wagner $\mathrm{C}$, et al. Adverse events and potentially preventable deaths in Dutch hospitals. Results of a retrospective patient record review study. Qual Saf Health Care 2009;18:297-302.

10. Zegers $\mathbf{M}$, de Bruijne MC, Wagner $\mathrm{C}$, et al. Design of a retrospective patient record study on the occurrence of adverse events among patients in Dutch hospitals. BMC Health Serv Res 2007;7:27.

11. Reason J. Human error. Cambridge: Cambridge University Press, 1990.

12. van Vuuren W, Shea CE, van der Schaaf TW. The development of an incident analysis tool for the medical field. Eindhoven: Eindhoven University of Technology, 1997.

13. van der Schaaf TW, Habraken MMP. PRISMA-Medical: a brief description. Eindhoven: Eindhoven University of Technology, 2005. 
14. Rasmussen J. Skills, rules and knowledge: signals, signs and symbols and other distinctions in human performance models. IEEE Trans Syst Man Cybern 1983;13:257-66.

15. Wilson RM, Harrison BT, Gibberd RW, et al. An analysis of the causes of adverse events from the Quality in Australian Health Care Study. Med J Aust 1999:170:411-15

16. Neale G, Woloshynowych M, Vincent C. Exploring the causes of adverse events in NHS hospital practice. J $R$ Soc Med 2001;94:322-30.

17. Davis $\mathbf{P}$, Lay-Yee R, Briant R, et al. Adverse events in New Zealand public hospitals 2: preventability and clinical context. N Z Med J 2003;116:U623.
18. Neale G, Chapman EJ, Hoare J, et al. Recognising adverse events and critical incidents in medical practice in a district general hospital. Clin Med 2006;6:157-62.

19. NVZ, Orde van medisch specialisten, LEV et al. Patient safety program Prevent harm, work safely in Dutch hospitals (in Dutch), Utrecht 2007.

20. Sherman H, Castro G, Fletcher $\mathbf{M}$, et al. Towards an international classification for patient safety: the conceptual framework. Int J Oual Health Care 2009:21: 2-8.

21. Runciman $\mathbf{W}$, Hibbert $P$, Thomson $R$, et al. Towards an international classification for patient safety: key concepts and terms. Int J Qual Health Care 2009;21:18-26.

\section{APPENDIX}

Examples of adverse events (AEs) and their causes and potential prevention strategies

\begin{tabular}{|c|c|c|c|}
\hline Description of AE & Main causal factors & Subcategories of $\mathrm{T}, \mathrm{H}$ and $\mathrm{O}$ & Prevention strategies \\
\hline $\begin{array}{l}\text { Leaking undersized intravascular aortic stent, } \\
\text { resulting in readmission and endovascular } \\
\text { reintervention }\end{array}$ & Technical, human & Materials (TM), intervention (HRI) & $\begin{array}{l}\text { Technology/equipment, training, } \\
\text { procedures }\end{array}$ \\
\hline $\begin{array}{l}\text { Inadequate planning operation (ankle fracture) } \\
\text { resulting in extended length of stay }\end{array}$ & Organisational & Management priorities (OM) & Procedures, motivation, staffing \\
\hline $\begin{array}{l}\text { Pneumonia after thoracotomy, requiring artificial } \\
\text { respiration and treatment with antibiotics }\end{array}$ & Other & - & - (not preventable) \\
\hline $\begin{array}{l}\text { (Blood) infusion rate too high in patient known } \\
\text { with heart failure and gastrointestinal bleeding, } \\
\text { resulting in respiratory insufficiency and } \\
\text { contributing to death }\end{array}$ & Human & $\begin{array}{l}\text { Knowledge (HKK), qualification (HRO), verification } \\
\text { (HRV) }\end{array}$ & Evaluation \\
\hline $\begin{array}{l}\text { Not-indicated surgery of adrenal gland (lung } \\
\text { metastases) resulting in needless suffering, } \\
\text { respiratory insufficiency and early death }\end{array}$ & Human, organisational & $\begin{array}{l}\text { Knowledge (HKK), coordination (HRC), knowledge } \\
\text { transfer (OK) }\end{array}$ & $\begin{array}{l}\text { Procedures, information and } \\
\text { communication, evaluation }\end{array}$ \\
\hline Persistent haemorrhage of wound feeding probe & Patient related & - & - (not preventable) \\
\hline $\begin{array}{l}\text { Missed diagnosis pulmonary embolism, } \\
\text { contributing to death }\end{array}$ & Human & Knowledge (HKK), violation (V) & $\begin{array}{l}\text { Training, procedures, quality assurance/ } \\
\text { peer review }\end{array}$ \\
\hline $\begin{array}{l}\text { Lens remains left behind after lens extraction, } \\
\text { requiring resurgery }\end{array}$ & Human & Intervention (HRI) & Training, quality assurance/peer review \\
\hline
\end{tabular}

\footnotetext{
*For explanation of causal factors, see table 1.
} 